\title{
Zwingli als sozialpolitischer Denker
}

\author{
von Arthur Rich \\ Professor an der Universität Zürich
}

Dite Ausgangsfrage

Huldrych Zwingli war seinem Naturell nach unzweifelhaft ein politischer Kopf. Von einem gegnerischen Zeitgenossen wurde er als Bürgermeister, Schreiber und Rat in einer Person bezeichnet ${ }^{1}$. Wenn auch die neuesten Forschungen das so nicht bestätigt haben, ist daran doch richtig, daß der Reformator durch sein ganzes, früh abgebrochenes Leben eine politisch höchst engagierte Rolle spielte ${ }^{2}$. Schon als Meßpriester zu Glarus hat er sich in der Gefolgschaft des berühmten Humanisten Erasmus von Rotterdam im Kampf gegen das Reislaufen und Pensionenwesen, ja den Krieg überhaupt, dermaßen exponiert, daß er das Feld räumen mußte ${ }^{3}$. Auch bei seiner Berufung ans Großmünster nach Zürich im Spätjahr 1518, die äußerlich den Beginn seiner Reformation in die Wege leiten sollte, standen, und zwar gehörig, politische Motive mit im Spiel ${ }^{4}$. So könnte man weiterfahren bis ans Ende seines Lebens, das ja, in einem prägnanten Sinne, auch ein politisches Ende war. Keiner unter den Reformatoren - von Luther ganz zu schweigen - hat so direkt ins große politische Geschehen eingegriffen wie Huldrych Zwingli. Das sei gesagt, ohne damit vorschnell ein Werturteil zu fällen. Doch stellt sich hier die Frage, wie seine politische Rolle mit derjenigen des religiösen

1 Johannes Salat, Chronik der Schweizerischen Reformation von deren Anfängen bis und mit 1534, in: Archiv für die Schweizerische Reformationsgeschichte, Bd. I, Solothurn 1868, S. 43. - Zur Beurteilung des Salatschen Zwinglibildes siehe die gründliche Studie von Fritz Büßer, Das katholische Zwinglibild von der Reformation bis zur Gegenwart, Zürich 1968, S. 59-78.

2 Joh. Caspar Mörikofer, Ulrich Zwingli nach den urkundlichen Quellen, Bd. I, Leipzig 1867, S. $130 \mathrm{ff}$, und in Anschluß an ihn die frühere Zwingli-Forschung überhaupt, meinte, daß der Reformator «der eigentliche Regent von Zürich» gewesen sei. Die Ergebnisse der jüngsten Forschungen von Martin Haas, Zwingli und der Erste Kappelerkrieg, Zürich 1965, Kurt Spillmann, Zwingli und die zürcherische Politik gegenüber der Abtei St. Gallen, St. Gallen 1965, und René Hauswirth, Landgraf Philipp von Hessen und Zwingli, Tübingen/Basel 1968, haben diese Behauptung als nicht zutreffend erwiesen. Zwinglis politischer Einfluß war bedeutend, doch keineswegs dominierend.

3 Darüber eingehend Oskar Farner, Huldrych Zwingli, Bd. II (Seine Entwicklung zum Reformator), Zürich 1946, S. 172-210 (Der Scharfmacher).

${ }^{4}$ Ebd., S. $294 \mathrm{f}$. 
Reformators zusammenstimme. Politik und Glauben werden ja zumeist als zwei grundverschiedene Welten aufgefaßt. Aber das gilt offenbar für Zwingli nicht. Er hat vereinigt, was man gewöhnlich trennt, ohne die Politik im Glauben oder den Glauben in der Politik aufgehen zu lassen. Wie ist das möglich? An Hand dieser Frage sollen jetzt die Grundlinien seines sozialpolitischen Denkens entfaltet werden.

\section{Der theologische Ort von Zwinglis sozialpolitischem Denken}

Wir stehen also vor der Ausgangsfrage, wie sich Politik und Glauben zueinander verhalten sollen. Das war nicht nur eine Frage $Z$ winglis, das war eines der großen Grundprobleme des Reformationszeitalters überhaupt. Es erhob sich in dem Augenblick mit zwingender Notwendigkeit, als man anfing, aus einer der Grunderkenntnisse der Reformation, nämlich daß allein das Wort Gottes in der Heiligen Schrift Norm und Maß des Glaubens sei, politische Folgerungen zu ziehen. In einem programmatischen und geschichtlich höchst folgenreichen Sinne geschah das zum ersten Male in den «Zwölf Artikeln der Bauernschaft zu Schwaben ${ }^{5}$ », Anfang 1525, zu Beginn des tragischen Bauernkrieges ${ }^{6}$. So heißt es gleich im Ingreß dieses berühmten Manifestes, $\mathrm{da} B$ die Bauern nichts anderes wollen, als das Evangelium hören und ihm gemäß leben. Unter «Leben» verstanden sie aber nicht nur die persönlich-private Existenz, sondern auch den politischen und sozialen Status in den gesellschaftlichen Verhältnissen. Auch er soll bestimmt sein durch die "göttliche Gerechtigkeit », die sich im Evangelium dem Glaubenden als Gottes Wort zuspricht. Deshalb begründen die Bauern ihre sozialpolitischen Forderungen mit Berufung auf die Bibel als Dokument der göttlichen Gerechtigkeit. Und deshalb sind sie auch bereit, wie ausdrücklich der zwölfte Artikel sagt, von «einer oder mehrerer» ihrer Forderungen abzustehen, wenn sie als unschriftgemä $B$ erwiesen werden können. Damit ist für die Bauern die Frage nach dem Verhältnis von Glauben und Politik, von Bibel und Gesellschaftsrecht entschieden. Beides hat sich zu decken; und wenn es sich nicht deckt, so können die bestehenden Rechtsverhältnisse in der Gesellschaft für sie auch keine bindende Kraft mehr haben. Aus dieser Überzeugung heraus beschritten sie den Weg der Rebellion.

5 Paul Joachimsen, Das Zeitalter der Reformation, in: Propyläen-Weltgeschichte, Bd.V, Berlin 1930, S. $103 \mathrm{ff.} \mathrm{Daselbst} \mathrm{auch} \mathrm{Faksimileabdruck} \mathrm{der} \mathrm{Zwölf} \mathrm{Artikel.}$

${ }^{6}$ Zum Gesamtaspekt siehe Günther Franz, Der deutsche Bauernkrieg, 7.Aufl., Bad Homburg 1965. 
Dem hat nun Martin Luther energisch widersprochen mit seiner «Ermahnung zum Frieden auf die zwölf Artikel der Bauernschaft in Schwaben ». Nicht daß er als «leidiger Fürstendiener », wie oft behauptet wird, sich schützend vor die von den Bauern angegriffenen Obrigkeiten gestellt hätte. Das Gegenteil ist wahr. Er ist mit den Machthabern ganz gehörig ins Gericht gegangen und hat sie dessen angeklagt, daß sie schinden und schatzen, um ihrem Prunk und Hochmut zu frönen, «bis [es] der arme gemeyne man nicht kan noch mag lenger ertragen ${ }^{7}$ ». Aber trotzdem sah er sich genötigt, gegen die in seinen Augen aufrührerischen Bauern vorzugehen. Wieder nicht, weil ihm deren Forderungen als verrückt erschienen wären. Er kann vielmehr sagen, etliche der zwölf Artikel seien «so billig und recht», daß sie den Fürsten vor «gott und der wellt den glympff nemen ${ }^{8} »$, diese also ins Unrecht versetzen. Der Grund liegt darum anderswo. Luther verargt den Bauern, daß sie als Christen sich selber Recht zu holen und ihre Forderungen als «christliche» auszugeben versuchen. "Darumb sage ich aber mal, Ich lasse ewr sachen seyn, wie gutt und recht sie seyn kan, weyl yhr sie aber selbs wollt verteydingen und nicht gewallt noch unrecht leyden, mugt yhr thun und lassen, was euch gott nicht weret, Aber den Christlichen namen, den Christlichen namen sage ich, den lasst stehen, und macht den nicht zum schanddeckel ewrs ungedultigem, unfridlichem, unchristlichem furnehmens ${ }^{9}$. » Auch darin liegt eine Antwort auf die Frage nach dem Verhältnis von christlichem Glauben und weltlicher Politik. Sie ist aber derjenigen der rebellierenden Bauern diametral entgegengesetzt. Stehen bei diesen Glauben und Politik, göttliche und irdische Gerechtigkeit, Reich Gottes und Weltreich ineinander, so tritt beides bei Martin Luther auseinander. Reich Gottes und Weltreich gehören zwei ganz verschiedenen Domänen an, wenn auch demselben Herrn verpflichtet. Denn Gott herrscht jeweils anders in den beiden ungleichen Reichen. Im Reiche Gottes oder geistlichen Reich regiert die Liebe, im weltlichen Reich dagen die «scharfe Barmherzigkeit", das heißt das Schwert, um eine drastische Wendung des Reformators zu gebrauchen. Darum muß man sich hüten, die beiden Reiche zu vermengen, wie es die Bauern tun. Das ist für Luther geradezu eine satanische Sache. "Dan der Teuffel will immerdar diese beyde reich in einander kochen und breuen ${ }^{10}$ », woraus nur Aufruhr und Revolution entstehen könne.

\footnotetext{
${ }^{7}$ Martin Luther, WA 18, 293 ${ }_{16-17}$.

${ }^{8}$ WA 18, 2984.

9 WA 18, 3149-14.

10 WA 47, $233_{42}-234_{1}$.
} 
Derart hat die Frage nach dem Verhältnis von Glauben und Politik, Reich Gottes und Weltreich im Reformationszeitalter eine schlechthin gegensätzliche Beantwortung gefunden. Wie steht unser Reformator zu der besagten, geschichtlich höchst bedeutungsschweren Kontroverse?

Wir befinden uns mit dieser einengenden Präzisierung unserer Ausgangsfrage an einem entscheidenden und deshalb zu großer Sorgfalt nötigenden Punkt. Zunächst ist $\mathrm{zu}$ sagen, daß für $\mathrm{Zwingli,} \mathrm{nicht} \mathrm{anders}$ als für Luther und auf ihre Weise auch die Bauern das Wort Gottes das «fundament" ist, «daruff alles gbüw sol gebuwen werden ${ }^{11}$ », mit dem also das Ereignis der Reformation steht oder fällt. Dabei will beachtet sein: Wort Gottes ist für Zwingli - und das auch wieder in Übereinstimmung mit Luther - nicht einfach das historische Wort, das sich in der Bibel findet, wie etwa die historische Lehre Platons in den Werken des großen Philosophen zu finden ist. Nein, Wort Gottes ist für ihn ein Geschehen, und zwar ein Geschehen, das jetzt und hier geschieht, frohe Botschaft, die den Menschen erleuchtet und zum Glauben bringt ${ }^{12}$. Er kann es darum in eins setzen mit Gott ${ }^{13}$ bzw. mit Christus ${ }^{14}$ selbst. Weil und sofern Gott selber zu uns kommt, geschieht sein Wort. «Also ist er waarlich uff der ban, sicht man an sinem wort. Wenn er das sendet, sol beBrung, volgen oder aber die straaff ist an der tür ${ }^{15}$. » "Wort Gottes » im Sinn des Reformators meint darum kein rhetorisches Gebimmel, sondern Erweis von heilschaffender Macht, die unsere ganze Existenz von Grund auf neu bestimmt ${ }^{16}$. Deshalb wird es für ihn gleichbedeutend mit «Reich Gottes» als dem Inbegriff von Gottes freimachender Herrschaft in der Welt. Schon in der Auslegung des 20. Artikels seiner SchluBreden (Anfang 1523) heißt es: «Das rych gottes ist nüt anderst denn das wort gottes ${ }^{17}$. " Und in der Erklärung des Matthäusevangeliums wird «regnum dei » als "der Handel des Wort Gottes» bezeichnet18. Das alles könnte Luther auch sagen und hat es ähnlich auch so gesagt. Trotzdem weiß sich $Z$ wingli gerade an diesem Punkt vom reformatorischen Bahnbrecher in Wittenberg geschieden, zumindest unterschieden. Für Luther ist die

$11 \mathrm{Z} \mathrm{I}, 338_{22-23}$.

${ }^{12} \mathrm{Z} \mathrm{I}, 372_{16-17}$ : O der frölichen botschafft, denn sy bringt mit ir ein liecht, das wir das wort war erkennend und gloubend, wie da oben vilvaltiklich bewärt ist. )

${ }_{13} \mathrm{Z} \mathrm{I}, 365_{31-32}$ : «... das wort gottes, das got selbs ist, erlüchtet alle Menschen.»

${ }_{14} \mathrm{Z} \mathrm{I}, 365_{24}$ : «... das wort gottes oder sun...»"

$15 \mathrm{Z} \mathrm{III,} \mathrm{42718-19.}$

${ }_{16} \mathrm{Z} \mathrm{I}, 353_{8-11}$.

17 Z II, 182,9-30.

$18 \mathrm{~S} \mathrm{VI} / 1,428$. 
Gegenwart des «regnum dei» Geschehnis des den sündigen Menschen gerechtsprechenden Evangeliums, mithin «ein unsichtbares Reich im Geist und inwendig in uns ${ }^{19} \%$. Es ist also niemals äußerlich. Und dem gerade widerspricht $Z$ wingli mit Entschiedenheit. Für ihn ist das Reich Gottes nicht nur innerlich, sondern auch äußerlich, «etiam externum ${ }^{20}$ ». Dessen kommende Gerechtigkeit hat also auch mit den äußeren Verhältnissen in der Welt zu tun, mit Staat, Gesellschaft, Politik. So schreibt er in seinem dem französischen König Franz I. gewidmeten Geleitwort zu «De vera et falsa religione Commentarius»: «Wer könnte leugnen, daß der Tag des Herrn gekommen ist? Nicht der letzte Tag, wo der Herr die ganze Welt richten wird, sondern (ein vorletzter Tag), da die gegenwärtigen Verhältnisse erneuert werden 21.» Dieser wichtige Passus, auf den noch zurückzukommen sein wird, weist nachdrücklich darauf hin, wie sehr das im geschehenden Wort schon jetzt wirkende Reich Gottes von Zwingli als eine die Welt verändernde Macht verstanden ist. Deshalb konnte er sich mit der Lehre von den beiden Reichen im Sinn des deutschen Reformators nicht befreunden ${ }^{22}$. Und insofern steht er in der Kontroverse zwischen Luther und den aufrührerischen Bauern diesen näher als jenem.

Wir haben damit eine erste Antwort auf die Frage bekommen, von der wir ausgegangen sind. Zwingli war Christ und Politiker zugleich; nicht weil er einfach in seinem politischen Naturell gefangen geblieben wäre, sondern weil er sich von der Macht des Wortes Gottes dazu getrieben sah. Der eigentliche Ort von $Z$ winglis sozialpolitischem Denken liegt darum auch nicht primär in seinem gleichsam angeborenen Interesse an den öffentlichen Dingen; er liegt im Wort Gottes, das «so lebendig, so krefftig » ist, daß es sich alle Dinge «glichförmig machen » will ${ }^{23}$, wie der Reformator schon 1522 sagt. Wer dies übersieht, wird $Z$ wingli nie verstehen.

19 WA 31/I, 505 ${ }_{36}-506_{2}$ : "regnum dei ... nempe in spiritu invisibile, <intra nos, non in externa observatione>. " Zu dieser Differenz zwischen Luther und Zwingli vgl. Siegfried Rother, Die religiösen und geistigen Grundlagen der Politik Huldrych Zwinglis, Erlangen 1956, S. $101 \mathrm{f}$.

${ }^{20} \mathrm{ZIX}, \mathbf{4 5 4}_{16-17}$ (in direkter Auseinandersetzung mit Luther): «Vult ergo Christus, etiam in externis modum teneri, eumque imperat; non est igitur eius regnum non etiam externum."

${ }^{21} \mathrm{Z} \mathrm{III,} 633_{16-18}$.

22 So bereits schon Erik Wolf, Die Sozialtheologie Zwinglis, in: Festschrift Guido Kisch, Stuttgart 1955, S. 178f., wie auch Gottfried W. Locher, Der Eigentumsbegriff als Problem evangelischer 'Theologie, 2.Aufl., Zürich 1962, S. 30.

$23 \mathrm{Z} \mathrm{I}, 353_{10-11}$. 


\section{Der Radikalismus des Wortes Gottes \\ (GÖTTLICHE GERECHTIGKEIT)}

Das sozialpolitische Denken Huldrych Zwinglis ist also aus dem ihm eigenen Verständnis des Wortes Gottes zu verstehen. Wort Gottes heißt Geschehnis des Reiches Gottes schon im «Jetzt», des Zukünftigen in der Gegenwart. Und zwar ist für ihn Reich Gottes samt der ihm zugehörigen göttlichen Gerechtigkeit, wie wir vorhin gesehen haben, auch äußerliche, nicht nur innerliche Wirklichkeit. Freilich auch innere Wirklichkeit. Soweit sie das ist, lehrt Zwingli von der Gerechtigkeit Gottes kaum viel anders als Martin Luther. Sie ist in diesem Falle die dem schuldigen Menschen geschenkte und ihn mit Gott versöhnende Gerechtigkeit ${ }^{24}$. Allein, auch als geschenkte Gerechtigkeit bleibt sie Forderung an den Christen. Darin liegt wieder ein Unterschied zu Martin Luther. Für diesen ist der Christenmensch von der Forderung des Gesetzes (natürlich des religiös-biblischen Gesetzes) frei. Auch $Z$ wingli weiß um die Freiheit vom Gesetz; doch versteht er das nicht in selber Weise. «Wir sind vom Gesetz nicht in dem Sinn befreit, daß wir das, was das Gesetz verlangt, nicht zu tun brauchten; denn das Gesetz ist Gottes unwandelbarer Wille... Wir sind in dem Sinne (von ihm) befreit: Wer liebt, tut alles freiwillig, auch das Schwerste ${ }^{25}$. \ Das Gesetz ist demnach, wie es hier verstanden wird, «keineswegs blo $ß$ jener Pädagoge, dessen einzige Aufgabe darin besteht, den Sünder in die Arme von Christus zu treiben ${ }^{26}$ ». Es ist primär, wie wir sahen, «ewiger Gotteswille ${ }^{27}$ » und somit gute Weisung an den Einzelnen wie an die Welt. Als solches ist es genau wie das Evangelium ein Wort des Lebens, das unsern Geist erquickt ${ }^{28}$. Darum kann auch das Gesetz bei Zwingli nicht im Lutherschen Sinne dem Evangelium entgegengesetzt sein ${ }^{29}$. Es ist vielmehr als gute Weisung Teil von ihm.

$24 \mathrm{Z} \mathrm{II}, 477_{24}$ : «An diser grechtikeit můssen alle menschen erligen; denn weleher ist so heylig, des hertz one anfechtungen und begird sye ? Also mag ouch keiner by got wonen; denn welcher by im wil wonen, můß one masen [Flecken] sin. Dis unser iamer und onmacht hat got gesehen und darüber erbarmt und mittel funden, damit sin grechtigheit versůnet wurde für uns, das wir by im wonen möchtind; und hat darumb sinen sun lassen mensch werden» usw.

${ }^{25} \mathrm{Z}$ III, $710_{3-9}$.

${ }^{26}$ So Heinrich Schmid in seiner ausgezeichneten Monographie: Zwinglis Lehre von göttlicher und menschlicher Gerechtigkeit, Zürich 1959, S. 121.

$27 \mathrm{Z} \mathrm{III,} \mathrm{7071:} \mathrm{«Lex} \mathrm{nihil} \mathrm{aliud} \mathrm{est,} \mathrm{quam} \mathrm{aeterna} \mathrm{dei} \mathrm{voluntas.»}$

${ }_{28} \mathrm{~S}$ VI/1, 599: "Nam ubi studium est iustitia, ibi gaudent homines, quum de iustitia fit mentio. »

${ }^{29}$ Heinrich Schmid, a.a.O., S. 124. 
Somit gehört zur Predigt des Evangeliums die Verkündigung der göttlichen Gerechtigkeit als Forderung an den Einzelnen wie an die gesellschaftliche Welt. Was aber meint hier "göttliche Gerechtigkeit»?

Unter ihr versteht der Reformator, kurz gesagt, die Gerechtigkeit, die Gott selber ist ${ }^{30}$ und die darum alles menschliche Recht, selbst das sogenannte «Naturrecht», transzendiert ${ }^{31}$. Sie kann also mit nichts verglichen werden, ist mithin analogielos und in solchem Sinne absolute Gerechtigkeit ${ }^{32}$. An ihr gemessen, erweist sich alle, auch die beste menschliche Gerechtigkeit als «ein arme, prästhaffte grechtigheit ${ }^{33}$ », "nit wirdig», «das man sy ein grechtigkeit nenne ${ }^{34}$ ».

Fragt man nach dem Inhalt der göttlichen Gerechtigkeit als Forderung an den Einzelnen wie an die gesellschaftliche Welt, so kann es letztlich nur eine Antwort geben, nämlich «Liebe». Wie Gott als der Gerechte seinem Wesen nach «Liebe» ist, so sollen es auch die Menschen, genauer die Christen, sein. Im Gebot der Liebe faßt darum Zwingli alle Forderungen der göttlichen Gerechtigkeit zusammen: "So sind alle vorgezelten gebot» - nämlich die einzelnen Forderungen der göttlichen Gerechtigkeit ${ }^{35}$ - «in den zwey fürnemsten gebotten verschlossen: Du solt dinen herren got lieb haben uß gantzen dinen hertzen, seel, gmůt und krefften, unnd dinen nächsten als lieb haben als dich selbs ${ }^{36}$. 》 Dieses Gebot ist nach der Meinung des Reformators ausgelegt in der Bergpredigt, der denn auch die meisten der von $Z$ wingli genannten Einzelforderungen der göttlichen Gerechtigkeit entnommen sind.

Ist nun dergestalt die göttliche Gerechtigkeit das Maß, an dem alles gemessen werden will, so liegt auf der Hand, daß dies zu einer radikalen Gesellschaftskritik führen mußte. Tatsächlich hat Huldrych Zwingli grundlegende Institutionen der Gesellschaft seiner, indirekt auch unserer Zeit von der göttlichen Gerechtigkeit her in Frage gestellt, wobei er scharf ins Zeug gefahren ist. So bestritt er schon im Jahre 1520 die Zulässigkeit des Zehnten nach dem göttlichen Recht ${ }^{37}$. Auch das Privat-

$30 \mathrm{Z}$ II, 475 17 : «... dann er ist die grechtigheit...»"

$31 \mathrm{Z} \mathrm{II,} \mathrm{4758-9}$ : "Got ist nit allein darumb grecht, das er eim ieden das sin (suum cuique!) gibt, als die mentschen die grechtigheit beschriben hand.» Deshalb ist auch die "grechtigheit gottes» erkennbar nur «an sinem eignen wort" (ebd., S. 4752r).

32 Siehe dazu vor allem Heinrich Schmid, a.a.O., S. $47 \mathrm{ff}$.

${ }^{33} \mathrm{Z} \mathrm{II}, \mathbf{4 8 5} 26-27$.

$34 \mathrm{Z} \mathrm{II,} \mathrm{48618-19.}$

35 Sie stehen in $\mathrm{Z} \mathrm{II}, 479_{20}-481_{14}$.

36 Z II, 482, $18-21$ (Mt. 22, 37-39).

37 Z VII, 272 $12-16$ : "Praepositus item noster virus quoddam effudit atque, ut memorabile esset, literis mandavit. Literae ad me sunt scriptae, quibus ille decimas iure divino constare dixit, contra quam (!) ego publice, latine tamen, non germanice, 
eigentum blieb von seiner Kritik nicht verschont. Dessen Grund liege keineswegs im Willen Gottes, sondern letztlich darin, daß wir von der Nächstenliebe abgekommen seien ${ }^{38}$. Alles gehöre im eigentlichen Gott. Und was «er uns fry gibt, das machend wir eigen ${ }^{39}$ », behaupten also wir als unser Privateigentum, worüber man absolut verfügen könne. Mit dem Eigentum eng verhängt ist das Problem der Geld- und Bodenzinse, die schon damals so viele Bauern in ärgste Schwierigkeiten brachten ${ }^{40}$. Dazu sagt der Reformator klipp und klar, Christus habe die Reichtümer gerechtermaßen für unrecht gehalten, "zů eim teil, daß wir eigen machend, das gottes ist, zum andern, daß wir, das gottes ist, darüber er aber uns laßt schaffner sin, nit nach sinem willen bruchend. Also sind ouch alle zins ungötlich ${ }^{41}$ ». Mit diesen wenigen Beispielen der Zwinglischen Gesellschaftskritik müssen wir uns jetzt begnügen.

Es dürfte sich von selbst verstehen, daß solcher Kritik an der vorhandenen gesellschaftlichen Ordnung eine revolutionäre Dynamik innewohnen mußte. Sie hat auch so gewirkt. Es ist vieles in Bewegung geraten unter dem Eindruck der Zwinglischen Predigt von der göttlichen Gerechtigkeit. Nicht nur religiös-kirchlich, auch politisch-sozial. Im Jahre 1522 läßt sich der konservative bernische Tagsatzungsbote, Kaspar von Mülinen, in einem freilich nicht untendenziösen Bericht an den Rat der Stadt Bern besorgt vernehmen, man solle beizeiten dem neuen Glauben in Zürich wehren, der den Umsturz aller Ordnung nach sich ziehen müsse. "Und hat die sach sich also ingerissen, daß unsere puren uf dem Land weder Zins noch zehenden mer wöllent geben ${ }^{42}$. » Tatsächlich sind derartige Weigerungen in nicht unbeträchtlicher Zahl vorgekommen, vorab bei geschuldeten Abgaben an geistliche Grundherrschaften ${ }^{43}$. Wie weit sie direkt auf Z winglis Kritik zurückgehen, ist natürlich schwerlich auszumachen. Denn man muß sich stets gegenwärtig

dixeram. » Vgl. dazu Theodor Pestalozzi, Die Gegner Zwinglis am Großmünsterstift in Zürich, Zürich 1918, S. $89 \mathrm{ff}$.

${ }^{38} \mathrm{Z} \mathrm{II}, 511_{2-8}$ : «Nun kundt [kommt] die schuld dahar, das wir das gebott gottes nit haltend: Du solt dinen nächsten als lieb haben als dich selbs. Denn wo wir das hieltind, so hulffe, der etwas für hat, von im selbs dem manglenden. So wir aber das nitt haltend, so sind die frücht und hab diser welt in der menschen eigenschafft [Eigentum] kummen, und haltend die inn, das got fry unverkoufft hat geben. »

${ }^{39} \mathrm{Z} \mathrm{II}, 51 \mathrm{l}_{12-13}$.

40 Vgl. Anm. 91.

$41 \mathrm{Z} \mathrm{II}, 515_{28}-516_{4}$.

42 Emil Egli, Actensammlung zur Geschichte der Zürcher Reformation 15191533, Zürich 1879, Nr. 370.

${ }^{43}$ Siehe dazu Walther Köhler in seiner Einleitung zu: "Wer Ursache gebe zu Aufruhr » usw., Z III, $362 \mathrm{f}$. 
halten, daß Zehnt- und Zinsverweigerungen im Vorfeld des großen Bauernkrieges schon ohnehin in der Luft gelegen haben. Allein, gesichert bleibt, daß sich damals mit Berufung gerade auf $Z$ winglis Lehre von der göttlichen Gerechtigkeit ein radikaler Flügel innerhalb der Zürcher Reformation gebildet hat, der nicht nur die Zins- und Zehntenzahlung, sondern überhaupt die gesellschaftliche Rechtsordnung - zumindest für die Christen - schlechterdings in Frage stellen wollte ${ }^{44}$. Es ist dies der Anfang der in vielem so sympathischen und auch oft arg verkannten Täufergruppen ${ }^{45}$, die, teils aus sachlicher Übereinstimmung, teils unter dem Zwang der Zeitumstände in mannigfache Verschlingungen mit der Bauernbewegung hineingerieten, obwohl die meisten unter ihnen im Grunde unpolitisch waren. Der Einfachheit halber werden wir sie fortan die "Radikalen» nennen ${ }^{46}$. Erst in harter Auseinandersetzung mit den «Radikalen » hat sich Zwinglis sozialpolitisches Denken zu seiner eigentlichen Höhe emporgebildet.

\section{Die Differenz zu den «Radikalen 》 (Menschliche Gerechtigkeit)}

Die «Radikalen» sind von Zwingli ausgegangen ${ }^{47}$. Ihr Radikalismus erweist sich darum als «wurzelverwandt 48 » mit der Radikalität des Reformators, wie sie in seiner Botschaft von der göttlichen Gerechtigkeit als der wahren Gerechtigkeit des Reiches Gottes zum Ausdruck kommt. Was beide eint, ist die Überzeugung, daß das Reich Gottes und mithin

\footnotetext{
44 Ebd.

45 Über die Anfänge der Täuferbewegung siehe Fritz Blanke, Brüder in Christo, Zürich 1955.

46 Diese Terminologie hat Emil Egli, Die Zürcher Wiedertäufer zur Reformationszeit, Zürich 1879, eingeführt. Ich nehme sie nicht ohne Bedenken auf. Denn sie könnte suggerieren, daß Zwingli unradikal gewesen wäre. In Wirklichkeit muß aber von einer Radikalität $Z$ winglis gesprochen werden, die sozialpolitisch weit effektiver war als der in dieser Hinsicht eher scheinbare Radikalismus jedenfalls der Zürcher Täufer. Anderseits ist es neuerdings auch umstritten, ob die erst seit Dezember 1923 auftretenden "Proto-Täufer» überhaupt mit den "Radikalen» identisch seien. Dazu siehe John H. Yoder, A Review and Discussion about: Siegfried Rother, Die religiösen und geistigen Grundlagen der Politik Zwinglis; Heinrich Schmid, Zwinglis Lehre von der göttlichen und menschlichen Gerechtigkeit, in: The Mennonite Quarterly Review, Vol. 135 (1961), S.79-88. - Ein gemeinsamer Wurzelgrund zwischen den Radikalen und den Täufern dürfte jedoch auf alle Fälle bestehen.

$47 \mathrm{Z} \mathrm{IV}, 208_{25}$ : «Sy sind von uns ußgangen: dann [ $=$ doch] sy warend nit uß uns » (nach 1. Joh.2, 19). Dgl. Z VI/I, 478.

${ }^{48}$ So Oskar Farner, Huldrych Zwingli, Bd. III (Seine Verkündigung und ihre ersten Früchte), Zürich 1954, S. 383.
} 
seine Norm, die göttliche Gerechtigkeit, nicht nur, wie bei Martin Luther, innerlich-geistig, sondern auch äußerlich-weltlich sei und daß demzufolge das ganze Leben, eben auch das äußerlich-weltliche, sich nach dieser Norm zu richten habe. Aber daraus sind von $Z$ wingli und den «Radikalen" ganz verschiedene Konsequenzen gezogen worden.

Die «Radikalen» haben nämlich daraus gefolgert, daß an die Stelle der bisherigen Ordnung, und zwar nicht nur derjenigen der Kirche, sondern auch der der bürgerlichen Gesellschaft schlechthin, die Ordnung der göttlichen Gerechtigkeit zu treten habe. Alles, was in Kirche und Gesellschaft keinen ausdrücklichen Grund im Evangelium als dem Offenbarungswort der göttlichen Gerechtigkeit besitze, müsse fallen: Zehnten, Zinse, Privateigentum, obrigkeitlicher Zwang, das letztere vor allem im Zusammenhang mit der Ablehnung der Kindertaufe ${ }^{49}$. Und sie sahen sich darin lediglich als die wahren Testamentsvollstrecker der zürcherischen Reformation ${ }^{50}$. Davon hat sich Zwingli entschieden distanziert, vor allem in seinen sozialpolitisch bedeutsamsten Schriften: «Von göttlicher und menschlicher Gerechtigkeit» und «Wer Ursache gebe zu Aufruhr usw. ». Warum hat er das getan?

Man hat den Grund schon oft in einer «Kapitulation» des Reformators vor den "praktischen Lebensrealitäten" sehen wollen ${ }^{51}$, die sich nun einmal dem Anspruch der göttlichen Gerechtigkeit nicht fügen. Daran ist so viel richtig, daß die Distanzierung $Z$ winglis von den «Radikalen» mit einer andern Wertung der gesellschaftlich-staatlichen Wirklichkeit zusammenhängt. Doch der eigentliche Grund liegt tiefer. Er ist theologischer Natur.

An derselben Stelle, wo Zwingli eingesteht, daß die «Radikalen» von ihm ausgegangen seien, sagt er nämlich mit Betonung, daß sie nicht bei ihm geblieben wären ${ }^{52}$. Demnach sind sie von ihm abgewichen. Worin ist der entscheidende Punkt ihrer Abweichung zu sehen?

Er ist darin zu sehen, daß sie aus der Sicht des Reformators nicht im rechten Sinn zu unterscheiden wissen zwischen evangelischer Forderung

49 So wieder Walther Köhler an dem in Anm. 43 angegebenen Ort.

50 In der Frage der Kinder- bzw. Erwachsenentaufe, die bald alles übrige überspielte, ist das direkt zu belegen. Sowohl Felix Manz in seiner Konrad Grebel zugeschriebenen «Protestation und Schutzschrift an den Rat von Zürich» (ZIII, $371_{25 \text { P. }}$. und $372_{6}$ ) wie Balthasar Hubmaier in seinem «Gespräch von Friedberg » (Z IV, 228, Anm. 21) berufen sich auf $Z$ wingli.

${ }^{51}$ So Walther Köhler, Die Geisteswelt Ulrich Zwinglis, Christentum und Antike, Gotha 1920, S. 124, dgl. abgeschwächt, Huldrych Zwingli, Leipzig 1943, S. 150. Dem schließt sich u.a. an: Paul Meyer, Zwinglis Soziallehren, Linz a.d.D. 1921.

52 Z IV $208_{26-27}$ : "Dann wärind sy uB uns gewesen, so wärind sy by uns bliben.» 
und bürgerlichem Gesetz, zwischen «göttlicher» und «menschlicher» Gerechtigkeit. Denn würden sie das tun, sie könnten nicht die göttliche Gerechtigkeit in einem direkten Sinne an die Stelle der menschlichen setzen wollen.

Die göttliche Gerechtigkeit, so sahen wir bereits, ist in ihrem Wesen Liebe. Das unterscheidet, ja trennt sie von allem menschlichen Recht, auch von dessen höchsten Formen, weil dieses nie Liebe ist noch Liebe sein kann. Liebe ist nun zwar gefordert: «Du sollst deinen Nächsten lieben wie dich selbst!» Allein, Liebe läßt sich nicht erzwingen. Nur die göttliche Gerechtigkeit, und das heißt jetzt Gott allein, der diese Gerechtigkeit selbst ist, kann Gottes Gerechtigkeit somit zur Herrschaft bringen. Der "Anhub» muß von Gott her kommen ${ }^{53}$. Das war immer Zwinglis volle Überzeugung, seitdem er zur reformatorischen Grunderkenntnis durchgestoßen war ${ }^{54}$. Darum war er, den man so gern als unbekümmerten Draufgänger zeichnet, jeder Zwängerei im Blick auf das Letzte, auf die Gerechtigkeit Gottes oder die Wahrheit des Glaubens, zutiefst abhold ${ }^{55}$. Freilich, wir haben das bereits vernommen, sah Zwingli im Ereignis der Reformation Einbruch des Gottesreiches, «Tag des Herrn ${ }^{56}$ ». Aber eben - man erinnere sich - nicht den letzten Tag des Herrn, da Gottes Gerechtigkeit und Wahrheit alle Welt durchstrahlt. Dieses «Letzte» läßt sich nicht erzwingen. Ja, Zwingli kann sich, allerdings in einem anderen Zusammenhang, sogar fragen: «Wie, wenn Gott die Verbreitung dieses Lichtes noch nicht wünschte ${ }^{57}$ ? » Er steht hier vor uns als ein Mensch, «der sich seine Angriffslust durch den Blick auf das göttliche «Nochnicht) zügeln läßt58》. So weiß er, trotz dem «Schon-jetzt» der göttlichen Gerechtigkeit als Antrieb in Mensch und Welt um das "Nochnicht» und damit um das «Immer-noch» des Unrechts, des Bösen, des «Prästen», wie er gerne sagt, sowohl unter «Gottlosen ${ }^{59}$ » als auch unter «Christen ${ }^{60}$ ». Die «Radikalen» verkennen das «Noch-nicht»; sie sind im Sprung auf das «Schon-jetzt». Für sie ist darum die fraglos frag-

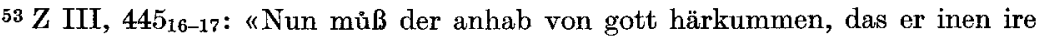
hertzen erlüchte, das sy inn erkennind und fürchtind.»

54 Siehe dazu Arthur Rich, Die Anfänge der Theologie Huldrych Zwinglis, Zürich 1949, S. 96-104.

${ }^{55}$ Vgl. dazu Fritz Blanke, Zwinglis Hauptmotive in der Abendmahlslehre und das Neue Testament, in: Zwingliana, Mitteilungen zur Geschichte Zwinglis und der Reformation, hg. vom Zwingli-Verein Zürich, Bd. V, 1929-33, S. 187.

56 Siehe S. 71 und Anm. 21.

$57 \mathrm{Z} \mathrm{V}, 562_{12-13}$.

${ }^{58}$ Fritz Blanke, ebd.

59 Z. B. Z II, $483_{16-24}$.

60 Z.B. Z III, $714_{24-29}$. 
würdige Ordnung der Gesellschaft - wir würden heute sagen das «Establishment» - schlechthin abgetan. Es gilt nur noch die "göttliche», nicht mehr die «menschliche» Gerechtigkeit. Denn die Liebesordnung kommt ohne alle festen Institutionen aus.

Für Zwingli heißt das nun, und zwar mit Recht, Eliminierung der Gerechtigkeit aus der faktischen Gesellschaft. Denn die faktische Gesellschaft ist, wie er meint, eine Gesellschaft von «Aufrührern», die in die Schranken gewiesen werden müssen. Wenn unser Reformator von «Aufrührern " redet, dann meint er jedoch nicht, wie Martin Luther, in erster Linie die rebellischen Bauern oder nonkonformistischen Täufergruppen. Er meint zwar diese auch, doch die «wahren Aufrührer» sind für ihn die - und das ist jetzt in unser heutiges Deutsch übertragenes Zitat -, «die zwar nicht so genannt sein wollen, dafür aber andere mit dieser Titulatur bewerfen ${ }^{61} »$. Und das sind erstens «die hohen bischoff ${ }^{62}$ », zweitens «die übrigen zal der widerbefftzenden pfaffen, münchen, nonnen, voruß der äbten ${ }^{63}$ » und drittens «die fürsten, gewaltigen und rychen diser welt ${ }^{64} \gg$. Das ist eine höchst schockierende Enumeration, die uns ahnen läßt, wen $Z$ wingli heute zu den eigentlichen Aufrührern rechnen müßte. Es sind diejenigen, die Macht haben, ihre Macht jedoch im eigenen, sei es persönlichen oder klassenmäßigen Interesse mißbrauchen, so andere unterdrücken, ausnutzen und ihren Zielen gefügig machen. Dieser Aufruhr von oben führt zum Aufruhr von unten als dessen unvermeidliche Reaktion ${ }^{65}$. Der Aufruhr gegen die eigentlichen Aufrührer ist so für den Reformator verständlich, aber er ist trotzdem nicht gutzuheißen. Denn beides, tyrannische Gewalt wie antityrannische Gegengewalt, ist letztlich Ausdruck von Gewaltverfallenheit. Und "wär nun [= nur] uff gwalt sicht (so oder so), der ist ye [immer] ufrürisch 66 ”. Solche Gewaltverfallenheit kann allein das Recht in Schranken halten, genauer das erzwingbare Recht, also die menschliche Gerechtigkeit. «Denn sölte uns die arme grechtigheit erst ouch entgon ${ }^{67}$, wie uns die götlich entgangen ist, so wäre mentschlich geselschafft nüt anderst dann ein leben der unvernünff-

${ }^{61} \mathrm{Z}$ III, 412 $2_{18-19}$ : «Diser teil wirt sagen von den warlich ufrůrigen, die doch deß nit namen, sunder uff ander wellend gelegt haben."

$62 \mathrm{Z}$ III, $412_{20}$.

${ }^{63} \mathrm{Z} \mathrm{III}, 420_{7-8}$.

$64 \mathrm{Z} \mathrm{III,} 423_{14-15}$.

65 Z III, 416 : «... das üch ouch gwalt wirt anthủn, glych wie ir gwalt thủnd.»

${ }^{66} \mathrm{Z}$ III, $420_{17-18}$.

67 Zwingli will damit sagen, daß der Mensch die göttliche Gerechtigkeit nicht zuhanden hat (sie ist ja Wirkung Gottes), wie er das menschliche Recht zuhanden haben kann. 
tigen thieren: Welcher stercker, dem wäger ${ }^{68}$.» Wer mehr Macht, dem mehr Recht.

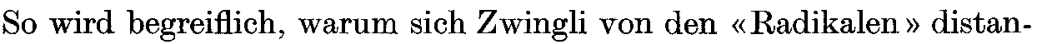
ziert. Er will die menschliche Gesellschaft - in der Zeit des Vorletzten nicht dem Faustrecht überlassen. Das wäre aber unweigerlich der Fall, wenn mit Berufung auf die göttliche Gerechtigkeit die menschliche, das heißt das erzwingbare Recht, mißachtet, ja selbst beseitigt würde. Göttliche oder menschliche Gerechtigkeit, das ist in der Zeit vor dem letzten Tag eine falsche Alternative. Es muß heißen: Göttliche und menschliche Gerechtigkeit.

In diesem Sinne also sieht sich Zwingli fortan genötigt, gegen die «Radikalen » Recht und Notwendigkeit der menschlichen Gerechtigkeit ins Feld zu führen. Die menschliche Gerechtigkeit ist notwendig, weil sie zeitliche Not wendet. Sie weist, sofern sie nicht entartet ist, den Gewalttäter in die Schranken. Sie wehrt dem tief eingewurzelten Eigennutz, der andauernd das Recht des Nächsten verletzt. Würden wir die göttliche Gerechtigkeit halten, die im Liebesgebot gipfelt, es gäbe freilich weder «roub noch diebstal», das heißt, weder Ausbeutung noch widerrechtliche Aneignung von Eigentum, das einem nicht gehört. "So aber das nit ist, so gibt got das nidrest gebott, das menschliche gselschafft ... erlyden mag: Du solt nit stelen 69 !» Was von diesem «niedrigsten » Gebot gilt, gilt auch von allen andern, aus denen sich die Summe menschlicher Gerechtigkeit zusammensetzt ${ }^{70}$. Derart, also in einem indirekten Sinne, ist auch die menschliche Gerechtigkeit als das in Gesellschaft und Staat gültige Recht von Gott geboten. «Wiewol dise menschliche grechtigheit nit wirdig ist, das man sy ein grechtigkeit nenne, so man sie gegen der götlichen grechtigheit besicht, so hat doch gott sy ouch gebotten ", aber erst, wie die zitierte Stelle weiterfährt, auf unsern Ungehorsam gegenüber dem Anspruch der göttlichen Gerechtigkeit hin ${ }^{71}$. Sie ist also immer nur sekundäres oder besser relatives Recht inmitten einer relativen Welt, die noch nicht Reich Gottes ist. Innerhalb dieses Relativen ist allerdings die menschliche Gerechtigkeit Funktionsersatz für das Liebesgebot ${ }^{72}$, sofern sie den Menschen in seiner gesellschaftlichen

$68 \mathrm{Z} \mathrm{II}, 488_{1-4}$.

${ }^{69} \mathrm{Z} \mathrm{II}, 490_{10-12}$.

${ }^{70} \mathrm{Z} \mathrm{II}, 487_{14}-493_{3}$ konfrontiert Zwingli an zehn Punkten die niedrigen Gebote der menschlichen Gerechtigkeit mit dem hohen Gebot der göttlichen.

71 Z II, 48618-21.

72 Vgl. Z II, 492 $10-12$ : «Das wir das einig gebott «Du solt dinen nächsten ebnen als lieb haben als dich selbs ? nit haltend, darus entspringend alle andre gebott, die den nächsten betreffend.» 
Existenz vor willkürlichen Übergriffen, zumal den Machinationen der Mächtigen, schützt. Und darum gerade steht nicht in funktionalem Widerspruch zur göttlichen Gerechtigkeit, wer im gesellschaftlichen Bereich die menschliche Gerechtigkeit einhält, auch wenn sich diese mit jener materiell nicht deckt. Das gerade will Huldrych Zwingli den «Radikalen ", die die menschliche Gerechtigkeit im Grund verachten, auf die Seele binden.

Doch nicht die «Radikalen» allein sind die Zielscheibe des Reformators in seinen sozialpolitischen Hauptschriften, wie er immer wieder mißverstanden wird. Schon der vorherige Hinweis auf die "wahren Aufrührer» macht das deutlich. Es gibt noch eine andere Front, an der $Z$ wingli nicht minder heftig streitet. Gemeint sind diejenigen, die ihm wohl darob Beifall klatschen, daß er so energisch auf die Respektierung der geltenden bürgerlichen Gesetze drängt, die aber vom Anspruch der göttlichen Gerechtigkeit nichts wissen wollen, jedenfalls dann nicht, wenn er gegen ihre eigenen Interessen geht. Hauptsache für sie ist, daß mit Entschiedenheit der einseitigen Brechung von rechtskräftigen Gesetzen oder gültigen privatrechtlichen Verträgen und mithin der willkürlichen Verweigerung von Zehnt- und Zinsverpflichtungen oder der Mißachtung von sonstigen Eigentumsrechten der Riegel geschoben wird. Alles andere ist überflüssig. Wollen die "Radikalen» die göttliche Gerechtigkeit ohne die menschliche, so wollen diese - man könnte sie die "Etablierten» nennen - die menschliche Gerechtigkeit ohne die göttliche. Dafür aber ist der Reformator erst recht nicht zu haben.

Eben in derselben Schrift, da Zwingli den «Radikalen » die Notwendigkeit der menschlichen Gerechtigkeit im relativen Bereich der Gesellschaft vor Augen führt, spricht er auch davon, daß man unter allen Umständen die göttliche Gerechtigkeit zur Sprache bringen müsse. «Die götlichen grechtikeit sol man one underlaß allen menschen offnen [das heißt kundmachen] und predigen und die hut ee verlieren, ee man sich von dero predigen und ußkünden lasse tringen ${ }^{73}$.» Und warum? Nicht um die menschliche Gerechtigkeit in den gesellschaftlichen Verhältnissen schließlich doch noch durch die göttliche zu verdrängen, sondern um klarzumachen, daß Gott unendlich mehr fordert als bürgerliche Rechtlichkeit und Wohlanständigkeit. Dies alles zählt im Grunde für den Reformator wenig. «So nun einer nit stilt, ist er fromm vor den menschen... er ist aber by got ein schelm; denn er hat die begird und anfechtung zů frömbden gůt vilicht größer dann einer, der gestolen hat. [Denn]noch wirt der dieb gehenckt, darumb, das er ein wüssenlicher dieb ist. Und der

${ }^{73} \mathrm{Z} \mathrm{II,} 493_{27-29}$. 
gotsschelm, der vil gytiger ist über zytlich gůt, der ist für einen frommen verrůmpt [ = bekannt], darumb, das er nit ußgebrochen hat mit der that, noch $[=$ doch $]$ ist er vor got nit fromm ${ }^{74}$.» Und das besagt: «Wir sind vor got all schelmen 75 », auch wenn man uns in Hinsicht auf die menschliche Gerechtigkeit oder bürgerliche Wohlanständigkeit nichts vorwerfen kann. Denn es bleibt dabei: Mag das Privateigentum, und mögen viele andere Dinge vor dem Forum der menschlichen Gerechtigkeit noch so rechtlich sein, vor Gott gilt das nicht. «Welcher nun nit wůcher [das heißt nicht Geld auf Zins!] gibt, ist deßhalb vor den mentschen fromm... aber vor got ist er dennocht nit fromm, er verkouffe denn all sin hab und geb sy den armen. Thüt das gheyner, so ist ouch gheiner nach der göttlichen grechtigheit fromm ${ }^{76}$. " So weit also geht Huldrych Zwingli. Keine Rede davon, daß er die Radikalität des Wortes Gottes auch nur im mindesten durch die menschliche Gerechtigkeit abschwächen würde. «Die mit dem menschlichen Verhalten unvereinbaren Forderungen der göttlichen Gerechtigkeit bleiben für alle bestehen, unerschütterlich, absolut. An ihnen läßt der Prediger am Großmünster keine Abstriche und keine Kompromisse zu ${ }^{77}$. » Freilich, um es nochmals zu unterstreichen: Das alles will der Reformator nicht als unmittelbares gesellschaftliches Gesetz verstanden wissen, wie die "Radikalen », sondern zunächst als persönliche Forderung an den einzelnen Menschen. Sie ist insofern personethisch gemeint und soll aufdecken, daß der Mensch als vom Evangelium geforderte Person bei aller menschlichen Gerechtigkeit noch nicht gerecht ist vor Gott. Allein, sie ist doch wieder nicht nur so verstanden. In ganz bestimmter Weise, wie andeutend schon hervorgetreten ist, richtet sie sich auch an den Menschen als Gesellschaft. Damit tut sich eine neue Dimension auf. Und erst in ihr kommt der eigentlich originale Beitrag Zwinglis als sozialpolitischer Denker im Zeitalter der Reformation zum Zuge.

\section{Absolutes und Relatives - Das Verhältnis von göttlicher UND MENSCHLICHER GERECHTIGKEIT}

Göttliche und menschliche Gerechtigkeit, so sahen wir, verhalten sich zueinander wie Absolutes und Relatives. Vor dem Absoluten ist das Relative nichtig. Darum kann der Reformator die menschliche Gerechtig-

${ }^{74} \mathrm{Z} \mathrm{II}, 484_{29}-485_{3}$.

${ }^{75} \mathrm{Z}$ II, $485_{5}$.

${ }^{76} \mathrm{Z} \mathrm{II}, 489_{22-26}$.

77 So Ernst Ramp, Das Zinsproblem, Eine historische Untersuchung, Zürich 1949, S. 77. 
keit immer nur «ein arme, prästhaffte grechtigheit 78 » nennen. In dieser Sicht der Dinge, die zwangsläufig zu radikaler Gesellschaftskritik herausfordert und von der vorhin einige Kostproben laut geworden sind, konvergiert die Radikalität Zwinglis mit dem Radikalismus der «Radikalen».

Für die "Radikalen» heißt dies aber, daß die menschliche Gerechtigkeit, weil sie in ihrer Substanz der göttlichen nicht entspricht, den Christenmenschen auch nicht mehr wirklich verpflichten könne. Ihre Gesellschaftskritik wird demzufolge absolut. Sie haben nur noch ein verneinend-distanziertes Verhältnis zur vorhandenen Gesellschaft mit ihrer immer bloß menschlichen Gerechtigkeit. So stehen sie gleichsam auf dem Standpunkt des «letzten Tages ${ }^{79}$ », vor dem alles Vorletzte verurteilt ist. Und im Grunde sind da, angesichts der unverkennbaren Tatsache, daß die vorhandene Gesellschaft nun einmal immer noch besteht, nur zwei Konsequenzen möglich: entweder die revolutionäre Errichtung der Theokratie oder dann der unrevolutionäre Auszug aus der Gesellschaft. Den ersten Weg ging Thomas Müntzer, den zweiten Weg der Zürcherische Täuferkreis.

Zwingli dagegen steht nicht auf dem Standpunkt des «letzten Tages». Es dürfte genugsam klar geworden sein, daß für ihn die Reformationszeit zwar auch als «Tag des Herrn", aber eben als ein nur vorletzter Tag des Herrn verstanden ist. Anders gesagt: Er verbleibt sozialpolitisch im Bereich des Relativen. Gesellschaftlich-staatliche Ordnung im Bereich des Relativen bzw. in der Zeit des Vorletzten gibt es allein auf dem Boden der menschlichen Gerechtigkeit. Verlieren wir die, so ist sozialpolitisch alles verloren. In dieser bestimmten Hinsicht nähert sich nun Huldrych Zwingli Martin Luther. Trotzdem denkt er in seinem sozialpolitischen Ansatz doch nicht lutherisch.

Er denkt nicht lutherisch, weil seine theologischen Voraussetzungen in eine andere Richtung weisen als die "Lehre von den beiden Reichen». Absolutes und Relatives, göttliches und weltliches Reich sind zwar auch

\footnotetext{
78 Siehe Anm. 33.

${ }^{79} \mathrm{Im}$ Blick auf Thomas Müntzer ist das eindeutig zu belegen. Vgl. seine «Fürstenpredigt » (Auslegung des zweiten Kapitels Daniels), Thomas Müntzer, Schriften und Briefe, kritische Gesamtausgabe, Quellen und Forschungen zur Reformationsgeschichte, Bd. XXXIII, Gütersloh 1968 (S. 242-263), die völlig beherrscht ist von der Vorstellung der angebrochenen Endzeit. Siehe vor allem S.255 f.: «Es ist dieser text Danielis also klar wie die helle sonne, und das werck geht itzdt im rechten schwangke vom ende des funfften reichs der welt" usw. - Dieses ekstatischeschatologische Endzeitbewußtsein trat im Zürcherischen Täuferkreis wohl zurück, doch fehlte es aber kaum. Von «einem rätselhaften vereinzelten Ausbruch endzeitlicher Erwartung» berichtet Fritz Blanke, Die Propheten von Zollikon, Mennonitische Geschichtsblätter, Jg. 9, 1952, S. 2-10.
} 
für ihn zu unterscheiden, aber nicht in einer Weise, die die Welt des Staates und der Gesellschaft herauslösen würde aus dem Forderungsbereich des Evangeliums von der göttlichen Gerechtigkeit. Diese Forderung bleibt bestehen, nicht nur an den Menschen als einzelne Person, sondern auch an den Menschen als Staat und Gesellschaft. Das drückt sich darin aus, daß die menschliche Gerechtigkeit, die das politischgesellschaftliche Leben regelt, nicht ihre eigenen, sei es natur- oder vernunftrechtlich ${ }^{80}$ begründeten Normen hat, noch auch positivistisch ihre eigene Norm sein kann. Die menschliche Gerechtigkeit hat vielmehr an der göttlichen, in der Heiligen Schrift enthüllten Gerechtigkeit ihre Norm, oder aber sie ist nicht wirklich menschliche Gerechtigkeit ${ }^{81}$. Dergestalt bleibt das Weltreich auf das Gottesreich, die menschliche Gerechtigkeit auf die göttliche bezogen.

Sozialpolitisch hat dies jetzt zur Folge, daß bei Zwingli an die Stelle einer absoluten Kritik des Relativen bzw. der gesellschaftlichen Rechtsverhältnisse, wie er sie vorfand, eine relative Kritik der Gesellschaft und ihrer Einrichtungen im Horizont des Absoluten tritt. Das verbindet sich mit einer Relativierung aller gesellschaftlichen Verhältnisse. Anders gesagt: Der Reformator läßt Staat und Gesellschaft mit ihrer bloß menschlichen Gerechtigkeit gelten; aber nur relativ, nicht absolut. Absolut gilt allein die göttliche Gerechtigkeit. Das eine ist in seinen sozialpolitischen Konsequenzen bereits hinreichend dargelegt worden. Das andere bedarf dagegen noch der Erhellung.

Nur relatives Geltenlassen der menschlichen Gerechtigkeit und ihrer gesellschaftlichen Institutionen besagt zweierlei: Es besagt fürs erste, daß die Institutionen des menschlichen Rechts keinen Absolutheitsanspruch geltend machen können, und fürs zweite, daß sie variabel und mithin veränderbar sein müssen. Beides tritt bei $Z$ wingli klar zutage. Das sei zunächst am Problem der staatlichen Gewalt bzw. der «Obrigkeit» dargelegt, wie man noch im Reformationszeitalter sagte.

Es war für Zwingli ausgemacht, daß staatliche Autorität als Garant der menschlichen Gerechtigkeit im gesellschaftlichen Zusammenspiel sein

${ }^{80}$ Zwingli kann zwar häufig vom "gsatzt der natur» reden (z. B. Z II, 32429). Aber sobald er es inhaltlich bestimmt, fällt es zusammen mit dem Liebesgebot als dem Inbegriff der göttlichen Gerechtigkeit, die die Heilige Schrift erschließt (Z II, $\left.324_{18-27}, 328_{1-4}, 492_{10-13}\right)$. Heinrich Schmid, a.a.O., S. $96 \mathrm{f}$. bezeichnet mit Recht das "gsatzt der natur" als die "gesetzliche Form» des Liebesgebotes. Darum muß auch das "gsatzt der natur" von ihr (sc. der Liebe) her gedacht und nach ihr hin ausgerichtet werden. Von einem selbständigen, abseits der Offenbarung faßbaren Naturrecht kann bei Zwingli kaum die Rede sein.

81 Siehe dazu Heinrich Schmid, a.a.O., S. 210-212. 
müsse. Aber ebenso stand es für ihn fest, daß solcher Autorität kein absoluter Charakter zukommen dürfe. Für ihn gibt es einen Souverän über der staatlichen Souveränität, eben die göttliche Gerechtigkeit, «die schnůr Christi ", kurzum das Liebesgebot. Allein, verrät sich darin letzten Endes nicht doch wieder Schwärmerei ? Kann man denn Staat und Gesellschaft auf die Liebe gründen? Entspricht das nicht ausgerechnet dem, was gerade auch die «Radikalen» wollten? - Zwingli nimmt diesen Einwand auf: «Was gadt die schnůr Christi die fürsten an», diese Norm, die doch kein Mensch einhalten kann, solange er in dieser Zeit lebt, er mag so heilig sein, wie er wolle 82 ? Und seine Antwort? Wir haben genau auf sie zu achten, denn sie gerade bringt den neuen Ton: "Nieman mag dem gebott gottes nachkummen. Ist gwüß.»Wir verstehen hier aber «das gebott gottes halten» nach seiner Weisung in dem Sinne handeln, daß ihm der Mensch möglichst gleichförmig wird ${ }^{83}$. Also: Die staatliche Obrigkeit hat keine Liebesordnung zu sein, das wäre Unsinn. Sie darf sich aber auch nicht mit der Ausübung der «scharfen Barmherzigkeit» zufriedengeben. Sie hat vielmehr im Relativen dem nachzueifern, was die Liebe will, nämlich aus dem Staat einen Ort zu machen, da das Leben wahrhaft menschlich wird. Und damit kommt bereits der humane Rechtsstaat in den Blick, wenigstens von weitem. Tut aber die Obrigkeit das nicht, bleibt der Staat pure Gewaltordnung, die sich nach ihren eigenen Machtgesetzen richtet, wird er somit absolut - wie es damals schon im Zug der Zeit gelegen hatte-, nun wohlan, dann tritt er aus dem Relativen heraus, und dann kann die Kritik an ihm auch nicht mehr bloß relativ sein. Sie wird in diesem Falle absolut werden müssen. Und so kommt es im zweiundvierzigsten Artikel von «Auslegen und Gründe der Schlußreden" zu jener Leitthese, die Luther sich nie zu eigen hätte machen können: «So sy aber [eben die Obrigkeit] untrülich und ußer der schnưr Christi faren wurdind, mögend sy mit got entsetzt [ = abgesetzt] werden ${ }^{84}$. »

Ähnlich verhält es sich beim Problem des Eigentums. Wie der staatlichen Autorität, so spricht Zwingli auch dem privaten Eigentum sein relatives Recht als Institution der menschlichen Gerechtigkeit zu. Er wendet sich gegen die «Radikalen», die nach dem Vorbild der jerusalemischen Urgemeinde ${ }^{85}$ die Gütergemeinschaft zum gesellschaftlichen

$82 \mathrm{Z} \mathrm{II,} \mathrm{3431-3.}$

83 Ebd., 3433-6.

84 Z II, 342. - Wie ernst Zwingli das meint, erhellt seine Bemerkung weiter unten: «Hettind die Jüdischen iren künig [gemeint ist der 〈Tyrann〉 Manasse] nitt also ungestraffet lassen mütwillen, hett sy got nit gestrafft » ( $\mathrm{Z} \mathrm{II,} \mathrm{34413-14).}$

85 Apg. 2, 42-47; 4, 32-37. 
Ordnungsprinzip schlechthin erheben wollen ${ }^{86}$. Doch ein absolutes Eigentumsrecht kennt $Z$ Wwingli wieder nicht. Auch darüber steht ein höherer Eigentümer ${ }^{87}$. Und daraus folgt die für das menschliche Recht grundlegende Eigentumsthese: «Du solt din zytlich gůt nit für din haben; du bist nur ein schaffner darüber.» Du sollst es unter die Armen teilen und es nicht denen geben, die keinen Mangel haben ${ }^{88}$. Damit ist die soziale Pflichtigkeit des Privateigentums als Weise des Gleichförmigwerdens mit dem Anspruch der Liebe proklamiert. Nur unter dieser Bedingung kann es Zwingli gelten lassen. Wieder ein Grundsatz menschlicher Gerechtigkeit von höchster Tragweite. Er weist in die Richtung auf eine sozial durchgestaltete Gesellschaft. Wo hingegen das private Eigentumsrecht sich absolut setzt, also seine soziale Pflichtigkeit mißachtet, wie das Zwingli vorab in den frühkapitalistischen Monopolgesellschaften seiner Tage - man denke etwa an die Fugger in Augsburg - verkörpert sah, da freilich wird seine Kritik auch wieder absolut und von maßloser Schärfe: «Dise eigenköffer [so nennt der Reformator die kapitalistischen Monopolinhaber] solt ein gantze Christenheit vertryben und abstellen glych als einen pundtschůch 89 ", das heißt wie eine Verschwörerbande.

Noch ein letzter Punkt ist hier der Sache halber heranzuziehen: die Zinsfrage, die den Reformator intensiv beschäftigt hat ${ }^{90}$. Andeutend ist bereits darauf verwiesen worden, daß sie im Zusammenhang mit der bäuerlichen Bodenverschuldung akut geworden ist. Auf dem Boden lagen damals große Lasten ${ }^{91}$. Sie bestanden zumeist in Erbzinsen (Abgaben an den Grundherrn) und in Geldzinsen (Leistungen auf Grundpfanddarlehen) ${ }^{92}$. Die Grundpfanddarlehen kamen in zwei Arten vor. Einmal als Gülten und dann als Renten. Gülten sind ablösbar, Renten dagegen nicht. Viele Gülten gingen dadurch in Renten über, daß sie, wenn der Schuldner nach Verlauf von drei bis vier Jahren sein Kündigungsrecht

${ }^{86} \mathrm{Z}$ III, 402 1-5 $_{\text {: }}$ "Hie sichstu [hier siehst du] ouch ... das uns gott die zämengeschütten gemeinschafft [= Gütergemeinschaft] nit gebüt» (wobei hier, wie der Kontext zeigt, $Z$ wingli das Gebot im Sinn von «nidere gsatzt» im Auge hat).

${ }^{87} \mathrm{Z}$ II, 515 $22-23$ : «Wir machend aber unser eigen, das gottes ist. »

$88 \mathrm{Z} \mathrm{II}, 451_{9-12}$; Z III, $45 \mathrm{l}_{10 \mathrm{ff}}$.

$89 \mathrm{Z} \mathrm{II}, 340_{34}$. Vgl. ferner Z III, $430_{5 \text { ff. }}$ und Z II, $297_{6-24}$.

${ }^{90} \mathrm{Zum}$ ganzen siehe die aufschlußreiche Arbeit von Ernst Ramp, a.a.O., S. 59-81.

91 Nach der gründlichen Untersuchung von Walter Claassen, Schweizerische Bauernpolitik im Zeitalter Ulrich Zwinglis, Sozialgeschichtliche Forschungen, Heft IV, Weimar 1899, S. 111, betrug die Belastung des bäuerlichen Privateigentums durch Erbzinse (Abgaben an den Grundherrn), Gülten (Zins auf ablösbare) und Renten (Zins anf unablösbare Grundpfandschulden) 19,5\% vom Wert des Landes exkl. Wald und Gärten.

${ }^{92}$ Dazu Walter Claassen, a.a.O., S. $82 \mathrm{ff}$. 
nicht nutzen konnte, der allgemeinen Tendenz des Mittelalters auf Festlegung und Vererblichung zeitweiliger Rechtsverhältnisse folgend, für die «Ewigkeit» fixiert wurden ${ }^{93}$. Derart nahmen die erblichen oder "ewigen" Zinsverpflichtungen überhand. Sie wurden zu fatalen Dauerbelastungen, wogegen die Bauern vornehmlich rebellierten.

Ein weiteres Malaise bestand darin, daß der Darlehensgläubiger einen Schuldner von Haus und Hof vertreiben konnte, wenn dieser, sei es wegen einer Mißernte oder eines sonstigen Unglücks, seiner Zinsverpflichtung nachzukommen nicht mehr imstande war. Das aber bedeutete nichts anderes als legalisierte Enteignung der Armen durch die Reichen. Und darin gerade sah Z wingli die größte Gefahr und schlimmste Kalamität in der herrschenden Zinsordnung der Zeit. "Sehend aber, wie es daby umb den armen gemeinen mann stand. Dero vertrybt man einen hütt, den andern morn, und ist ghein erbermd [= Erbarmen] in üch ${ }^{94} . »$ Eine Änderung der Verhältnisse war schwierig. Denn gerade auf diesen Zinsen gründete sich «das Einkommen der städtischen herrschenden Klassen, die dafür dem Staate größtenteils unentgeltliche Dienste leisteten ${ }^{95} »$. Der pure Umsturz der Rechtsordnung hätte deshalb alles aus den Fugen bringen müssen. Und das konnte der Reformator aus seiner Einsicht in die Notwendigkeit der menschlichen Gerechtigkeit so nicht wollen. Aber er hat auch hier nach Kräften relativiert. Diese Zinsordnung, so sehr sie für die herrschenden Rentnerschichten profitabel ist, darf nicht «ewig» sein. Sie soll auf die Länge vielmehr verschwinden. So hat er die Errichtung neuer «Zinskäufe», wie man damals die Grundpfanddarlehen nannte, scharf bekämpft sowie die erblichen Zinsverträge ablösbar machen wollen ${ }^{96}$. Für die Übergangszeit, das heißt bis zur Entschuldung und der damit verbundenen Beseitigung der Zinsen, gibt Zwingli summarisch zusammengefaßt folgende Weisungen:

"Wo die allgemeine Übereinkunft den Zinskauf zuläßt, da muß der Schuldner ihm korrekt nachkommen. Ein Zins von 5\% muß getragen werden, wenn dieser auch keine Grundlage in Gottes Wort hat. Falls jedoch der Zins höher als 5\% angesetzt wurde, ist die Obrigkeit verpflichtet, dem Schuldner beizustehen, um eine Reduktion des Zinsfußes auf $5 \%$ zu erwirken, und zwar auch dann, wenn der Zins mit obrigkeitlicher Bestätigung festgelegt worden war. Die Ablösung soll so durchgeführt werden, daß weder Gläubiger noch Schuldner unbillig belastet

${ }^{93}$ Ebd., S. 83.

${ }^{94} \mathrm{Z}$ III, $436_{24}-437_{1}$.

95 Walter Claassen, a.a.O., S. 112.

${ }_{96} \mathrm{Z}$ III, $45 \mathrm{I}_{16}-452_{25}$. 
werden. Um aber die Ablösung zu erleichtern, sollen Teilzahlungen bei größeren Beträgen gestattet sein, in der Weise, daß jederzeit das halbe Kapital zurückbezahlt werden darf... Dabei betont $Z$ wingli, daß bei dieser Maßnahme nicht stehengeblieben werden dürfe, denn dadurch sei nur der größte Unrat weggeschafft, wobei des Wüsten noch genug bleibe ${ }^{97}$. 》 Auch daran zeigt sich wieder, was es heißt, im Bereich des Relativen, also in der gesellschaftlichen Welt und ihrem Recht, in immer neuen Anläufen der «schnůr Christi» möglichst gleichförmig zu werden. Es heißt gerade nicht die konservativen Kräfte der Beharrung mobilisieren, wie $Z$ wingli traditionellerweise immer wieder ausgelegt worden ist ${ }^{98}$ : es heißt im Gegenteil die menschliche Gerechtigkeit unter dem revolutionären Antrieb der göttlichen unaufhörlich und in einer Weise verändern, daß humanere Formen des Zusammenlebens in Gesellschaft und Welt möglich werden ${ }^{99}$.

\section{Die entscheidende Entdeckung}

Mit dieser Grundkonzeption hat der Reformator für seine Zeit einen neuen Weg beschritten. Er selber nennt ihn einmal «media via ${ }^{100}$ ». Es wäre banal und käme einer Verharmlosung der Sache gleich, wenn man das im Sinn von "goldenem Mittelweg" oder dergleichen mehr verstehen wollte ${ }^{101}$. Im Bereich der dynamischen Spannung zwischen dem Absoluten und dem Relativen, der göttlichen und der mensehlichen Gerechtigkeit gibt es keinen bequemen, billigen, nach allen Seiten Rechnung tragenden Mittelweg. Es gibt da letztlich nur den mühsamen, steinigen und anfechtungsvollen Weg der immerwährenden, bußfertigen

${ }^{97}$ Ernst Ramp, a.a.O., S. 72. - Ramp bestreitet auch die weitverbreitete Meinung, "Zwingli habe einen sogenannten mäßigen Zins von $5 \%$ sanktioniert». Er wolle «zur Wahrung von Recht und Billigkeit diese 5\% nur als Übergangslösung bis zur gänzlichen Entschuldung zulassen».

98 Das ist ausgesprochen der Fall in der freilich längst überholten Schrift von Constantin von Kügelgen, Die Ethik Huldreich Zwinglis, Leipzig 1902, S. 67-95. Aber auch die umfassende und sonst so imponierende Darstellung P.J.V.Pollets (OP), Zwinglianisme, in: Dictionnaire de Théologie catholique, Bd. 15/2, Paris 1950, Sp. 3745-3928, neigt zu einer eher konservativ-traditionalistischen Interpretation der «idées sociales» Zwinglis, nicht ohne zu bemerken: "Zwingli serait sans doute devenu le Savonarole de Zurich, s'il n'y avait pas eu les anabaptistes» (Sp. 3902).

99 Ähnlich Heinrich Schmid, a.a.O., S. 212.

$100 \mathrm{~S} \mathrm{VI} / 1,237$.

101 Nach dieser Richtung tendiert Paul Meyer in seinem bereits angeführten Werk: Zwinglis Soziallehren. 
Wandlung des Menschen als Person wie als Gesellschaft auf das Absolute hin, was eben in der Sprache Zwinglis "glichförmig sin» mit dem Anspruch der göttlichen Gerechtigkeit bedeutet ${ }^{102}$. Das heißt nichts anderes, als daß der rechte Staat, die rechte Gesellschaft, die rechten sozialen Verhältnisse, kurzum die rechte menschliche Gerechtigkeit zur permanenten, sich stets neuen Möglichkeiten öffnenden Aufgabe des christlichen Glaubens werden muß ${ }^{103}$. Statt über die rebellischen Geister zu lamentieren, gilt es darum entschieden für strukturelle Wandlungen einzutreten, die im Interesse aller Benachteiligten in der Welt zu gerechteren politischen, sozialen und wirtschaftlichen Ordnungen führen können: die rechte Antwort auf die revolutionäre Herausforderung einer jeden Zeit. Gerade darin besteht die entscheidende Entdeckung Huldrych Zwinglis in seiner Auseinandersetzung mit Luther auf der einen und den "Radikalen» auf der andern Seite. Es handelt sich, kurz gesagt, um die Entdeckung der sozialethischen Verantwortung des Christen bzw. der Kirche und damit einer Radikalität, die im Ansatz ernsthafter und effektiver ist als der Radikalismus der «Radikalen». Denn die «Radikalen» blieben, weil ohne politisch praktikable Alternative - das Absolute kann nun einmal keine solche sein -, politisch wirkungslos. Sie endeten, als die theokratischen Träumereien ausgeträumt waren, damit, sich vom gesellschaftlichen Engagement zurückzuziehen und die Welt Welt sein zu lassen. Zwingli dagegen sah sich in seiner neuerkannten, aus dem Glauben stammenden sozialethischen Verantwortung dazu angetrieben, politisch reale Wege zu finden, um Gesellschaft und Welt tatsächlich zu erneuern. Mag er dabei auch in vielem gefehlt haben - und er hat gefehlt - und mag er oft genug als eben immer auch politisch denkender Christ in den alltäglichen Seilziehereien von der «media via» zwischen dem Absoluten

102 Vgl. Z III, 38310-14. - Vgl. dazu Jacques Courvoisier, Zwingli, A reformed theologian, Richmond 1963, S. 88 f., der die Dinge ähnlich sieht.

${ }^{103} \mathrm{Da}$ B eine derartige Konzeption gesellschaftlicher Verantwortung aus dem Glauben nicht auf den Nenner der "Theokratie» gebracht werden kann, versteht sich von selbst. Die Analyse der Grundstrukturen von Zwinglis sozialpolitisehem Denken deckt sich mit dem Befund der jüngsten historischen Zwingli-Forschung, wonach zur Zeit des Reformators in Zürich keine Theokratie bestanden haben kann. Siehe dazu neuestens den Forschungsbericht von Leonhard von Muralt, Zum Problem der Theokratie bei Zwingli, in: Discordia concors, Festgabe für Edgar Bonjour, Bd. II, Basel 1968, und ders., Die politischen Voraussetzungen der Reformation Zwinglis, in: Neue Zürcher Zeitung, 190.Jg., Nr. 4 (3. Jan. 1969/III), insbesondere auf S. 18. Dagegen Gottfried W. Locher, Huldrych Zwinglis Botschaft, Zwingliana, Bd. X, S. 598, der aber einen anderen Theokratiebegriff voraussetzt ("Leitung auch der öffentlichen Dinge durch den Geist Gottes"), als er hier verwendet wird. 
und Relativen in die Niederungen des bloßen Kompromisses abgeglitten sein - und das ist geschehen -, er bleibt doch ein Mann, der sich, das angriffige Wort Gottes im Rücken, mit den vorwärtsdrängenden Kräften in Kirche und Welt verschworen hat. Sich seinem Geist öffnen heißt deshalb heute, so wie er es damals tat, aus der Radikalität des Glaubens für die wahrhaftige Neuordnung von Kirche und Welt eintreten - allen Widerständen zum Trotz. "Dann warlich, warlich», Gottes Wort «wirt als [= so] gwüß sinen gang haben als der Rhyn; den mag man ein zyt wol schwellen, aber nit gstellen ${ }^{104}$.»

104 Z III, 488 $7-8$. 